\title{
LIMIT CYCLES FOR A CLASS OF THREE DIMENSIONAL POLYNOMIAL DIFFERENTIAL SYSTEMS
}

\author{
JAUME LLIBRE ${ }^{1}$ AND JIANG YU ${ }^{2}$
}

\begin{abstract}
Perturbing the system $\dot{x}=-y(1+x), \dot{y}=x(1+x), \dot{z}=0$, inside the family of polynomial differential systems of degree $n$ in $\mathbb{R}^{3}$, we obtain at most $n^{2}$ limit cycles using averaging theory of first order. Moreover, there are such perturbed system having at least $n^{2}$ limit cycles.
\end{abstract}

\section{Introduction AND STATEMENT OF THE MAIN RESUlts}

We perturb the system $\dot{x}=-y(1+x), \dot{y}=x(1+x), \dot{z}=0$ inside the class of polynomial differential systems of degree $n$ in $\mathbb{R}^{3}$. The unperturbed system has the straight line $x=0, y=0$ and the plane $x=-1$ fulfilled of singular points, and on each plane $z=\bar{z}=$ constant the flow is invariant. In fact, on every plane $z=\bar{z}$ the singular point $(0,0, \bar{z})$ is a center.

Theorem 1. We consider the family of systems

$$
\begin{aligned}
\dot{x} & =-y(1+x)+\varepsilon(a x+F(x, y, z)), \\
\dot{y} & =x(1+x)+\varepsilon(a y+G(x, y, z)), \\
\dot{z} & =\varepsilon(c z+R(x, y, z)),
\end{aligned}
$$

where $F(x, y, z), G(x, y, z)$ and $R(x, y, z)$ are polynomials of degree $n$ starting with terms of degree 2. Then there exists an $\varepsilon_{0}>0$ sufficiently small such that for $|\varepsilon|<\varepsilon_{0}$ there are systems (1) having at least $n^{2}$ limit cycles bifurcating from the periodic orbits of the system $\dot{x}=-y(1+x), \dot{y}=x(1+x)$, $\dot{z}=0$.

Theorem 1 improves the results of [1] where perturbing the system $\dot{x}=$ $-y, \dot{y}=x, \dot{z}=0$ inside the same class of polynomial vector fields the averaging method up to first oder only can obtain at most $n(n-1) / 2$ limit cycles. Preliminary results in this direction where obtained by Żoładek in $[5,6,7]$. His main result is that the number of limit cycles that he can

1991 Mathematics Subject Classification. 37G15, 37D45.

Key words and phrases. linear center, limit cycle, averaging theory, polynomial differential system. 
obtain from the periodic orbits of the center at the origin of the invariant plane $z=0$ is of the order a constant $n$. But we study the limit cycles bifurcating from the periodic orbits at any plane $z=$ constant, not only from the plane $z=0$. In this way we get that the number of limit cycles is $n^{2}$.

\section{Limit CYCLES VIA AVERAGING THEORY}

In few words we can say that the averaging method $[3,4]$ gives a quantitative relation between the solutions of some non-autonomous periodic differential system and the solutions of its averaged differential system, which is an autonomous one. The next theorem provides a first order approximation in $\varepsilon$ for the limit cycles of a periodic differential system, for a proof see Theorem 2.6.1 of Sanders and Verhulst [3] and Theorem 11.5 of Verhulst $[4]$.

Theorem 2. We consider the following two initial value problems

$$
\dot{x}=\varepsilon f(t, x)+\varepsilon^{2} g(t, x, \varepsilon), \quad x(0)=x_{0},
$$

and

$$
\dot{y}=\varepsilon f^{0}(y), \quad y(0)=x_{0},
$$

where $x, y, x_{0} \in D$ an open subset of $\mathbb{R}^{n}, t \in[0, \infty),|\varepsilon| \leq \varepsilon_{0}, f$ and $g$ are periodic of period $T$ in the variable $t$, and $f^{0}(y)$ is the averaged function of $f(t, x)$ with respect to $t$, i.e.,

$$
f^{0}(y)=\frac{1}{T} \int_{0}^{T} f(t, y) d t .
$$

Suppose: (i) $f$, its Jacobian $\partial f / \partial x$, its Hessian $\partial^{2} f / \partial x^{2}, g$ and its Jacobian $\partial g / \partial x$ are defined, continuous and bounded by a constant independent on $\varepsilon$ in $[0, \infty) \times D$ and $|\varepsilon| \leq \varepsilon_{0}$; (ii) $T$ is a constant independent of $|\varepsilon|$; and (iii) $y(t)$ belongs to $D$ on the interval of time $[0,1 /|\varepsilon|]$. Then the following statements hold.

(a) On the time scale $1 /|\varepsilon|$ we have that $x(t)-y(t)=O(\varepsilon)$, as $\varepsilon \rightarrow 0$.

(b) If $p$ is a singular point of the averaged system (3) such that the determinant of the Jacobian matrix $\partial f^{0} /\left.\partial y\right|_{y=p}$ is not zero, then there exists a limit cycle $\phi(t, \varepsilon)$ of period $T$ for the system (2) which is close to $p$ and such that $\phi(t, \varepsilon) \rightarrow p$ as $\varepsilon \rightarrow 0$.

(c) The stability or instability of the limit cycle $\phi(t, \varepsilon)$ is given by the stability or instability of the singular point $p$ of the averaged system (3). In fact, the singular point $p$ has the stability behavior of the Poincaré map associated to the limit cycle $\phi(t, \varepsilon)$. 


\section{Preliminary Results}

To prove Theorem 1 we shall need the following three lemmas which are proved in [2].

Lemma 3. For $i, j \in \mathbb{N}$, we define

$$
I_{i, j}=\frac{1}{2 \pi} \int_{0}^{2 \pi} \frac{\cos ^{i} \theta \sin ^{j} \theta}{1+r \cos \theta} d t
$$

Then $I_{i, j} \neq 0$ if and only if $j$ is even number.

Lemma 4. For $i, j \in \mathbb{N}$, and $j$ even, $I_{i, j}=\sum_{\substack{s=0 \\ s \text { even }}}^{j}(-1)^{s / 2}\left(\begin{array}{c}j / 2 \\ s / 2\end{array}\right) I_{i+s, 0}$.

Lemma 5. For $i \in \mathbb{N}$, we have

$$
\begin{aligned}
I_{i, 0} & =\frac{1}{2 \pi} \int_{0}^{2 \pi} \frac{\cos ^{i} \theta}{1+r \cos \theta} d t . \\
& =\frac{(-1)^{i}}{r^{i} \sqrt{1-r^{2}}}+\sum_{\substack{l=1 \\
l \equiv i(\bmod 2)}}^{i}(-1)^{l-1} 2^{l-i}\left(\begin{array}{c}
i-l \\
(i-l) / 2
\end{array}\right) r^{-l} .
\end{aligned}
$$

\section{Proof of Theorem 1}

Let

$$
\begin{aligned}
F(x, y, z) & =F_{2}(x, y, z)+F_{3}(x, y, z)+\cdots+F_{n}(x, y, z) \\
G(x, y, z) & =G_{2}(x, y, z)+G_{3}(x, y, z)+\cdots+G_{n}(x, y, z) \\
R(x, y, z) & =R_{2}(x, y, z)+R_{3}(x, y, z)+\cdots+R_{n}(x, y, z)
\end{aligned}
$$

be polynomials such that $F_{i}, G_{i}$ and $R_{i}$ are the homogeneous parts of $F$, $G$ and $R$ of degree $i$, respectively. In cylindrical coordinates $x=r \cos \theta$, $y=r \sin \theta, z=z$, system (1) in the region $r>0$ can be written as

$$
\begin{aligned}
\dot{r} & =\varepsilon(a r+\cos \theta F+\sin \theta G), \\
\dot{\theta} & =1+r \cos \theta+\frac{\varepsilon}{r}(\cos \theta G-\sin \theta F), \\
\dot{z} & =\varepsilon(c z+R) .
\end{aligned}
$$

Here and in what follows $F, G$ and $R$ will denote $F(r \cos \theta, r \sin \theta, z)$, $G(r \cos \theta, r \sin \theta, z)$ and $R(r \cos \theta, r \sin \theta, z)$, respectively. System (5) in the region $r>0$ is equivalent to system 
(6)

$$
\frac{d r}{d \theta}=\varepsilon \frac{a r+\cos \theta F+\sin \theta G}{1+r \cos \theta}+O\left(\varepsilon^{2}\right)
$$

$$
\frac{d z}{d \theta}=\varepsilon \frac{c z+R}{1+r \cos \theta}+O\left(\varepsilon^{2}\right)
$$

Let $D$ be an arbitrary ball of radius smaller than 1 centered at the origin of $\mathbb{R}^{2}$ and $\varepsilon_{0}$ be a positive number. Then, system (6) satisfies the assumptions of Theorem 2 if $\varepsilon_{0}$ is sufficiently small and $D$ is fixed. In order to apply the averaging theory to system (6) we have to compute the averaged functions

$$
\begin{aligned}
& f=f(r, z)=\frac{1}{2 \pi} \int_{0}^{2 \pi} \frac{a r+\cos \theta F+\sin \theta G}{1+r \cos \theta} d \theta \\
& g=g(r, z)=\frac{1}{2 \pi} \int_{0}^{2 \pi} \frac{c z+R}{1+r \cos \theta} d \theta
\end{aligned}
$$

For each $k=2, \cdots, n$ we write

$$
\begin{aligned}
& F_{k}(x, y, z)=\sum_{i+j+l=k} a_{i, j, l}^{k} x^{i} y^{j} z^{l}, \\
& G_{k}(x, y, z)=\sum_{i+j+l=k} b_{i, j, l}^{k} x^{i} y^{j} z^{l}, \\
& R_{k}(x, y, z)=\sum_{i+j+l=k} c_{i, j, l}^{k} x^{i} y^{j} z^{l} .
\end{aligned}
$$

Now using the notation introduced in Lemma 3, the averaged functions write as

$$
f=a r+\sum_{k=2}^{n} \sum_{i+j+l=k} r^{i+j} z^{l}\left(a_{i, j, l}^{k} I_{i+1, j}+b_{i, j, l}^{k} I_{i, j+1}\right)
$$

and

$$
g=g(r, z)=c z I_{0,0}+\sum_{k=2}^{n} \sum_{i+j+l=k} c_{i, j, l}^{k} r^{i+j} z^{l} I_{i, j}
$$


LIMIT CYCLES OF 3-DIMENSIONAL POLYNOMIAL DIFFERENTIAL SYSTEMS 5

Consequently, $f$ and $g$ are polynomials in the variables $r, z$ of degree $n$. Now we obtain by the lemmas

$$
\begin{aligned}
g & =c z I_{0,0}+\sum_{k=2}^{n} \sum_{m=0}^{k} r^{m} z^{k-m} \sum_{\substack{i+j=m \\
j \text { even } \\
\text { even }}} c_{i, j, k-m}^{k} I_{i, j} \\
& =c z I_{0,0}+\sum_{m=0}^{n} r^{m} \sum_{k=m}^{n} z^{k-m} \sum_{\substack{i=0 \\
m-i \text { even }}}^{m} c_{i, m-i, k-m}^{k} I_{i, m-i}- \\
& \sum_{m=0}^{1} r^{m} \sum_{m \leq k<2}^{m} z^{k-m} \sum_{\substack{i=0 \\
m-i \text { even } \\
m}}^{m} c_{i, m-i, k-m}^{k} I_{i, m-i} \\
& c z I_{0,0}+g_{1}(r, z)+g_{2}(r, z),
\end{aligned}
$$

with

$$
\begin{aligned}
g_{1} & =\sum_{m=0}^{n} r^{m} \sum_{k=m}^{n} z^{k-m} \sum_{\substack{i=0 \\
m-i \text { even }}}^{m} c_{i, m-i, k-m}^{k} \sum_{\substack{s=0 \\
s \text { even }}}^{m-i} d_{s, m-i} \frac{r^{-(i+s)}}{\sqrt{1-r^{2}}} \\
g_{2} & =\sum_{m=0}^{n} r^{m} \sum_{k=m}^{n} z^{k-m} \sum_{\substack{i=0 \\
m-i \text { even }}}^{m-i} c_{i, m-i, k-m}^{k} \sum_{\substack{s=0 \\
\text { seven }}}^{m} d_{s, m-i} \sum_{\substack{l=1 \\
i-l \text { even }}}^{i+s} e_{i+s, l} r^{-l}
\end{aligned}
$$

where

$$
\begin{aligned}
d_{s, m-i} & =(-1)^{s / 2}\left(\begin{array}{c}
(m-i) / 2 \\
s / 2
\end{array}\right) \\
e_{i+s, l} & =(-1)^{l-1} 2^{l-(i+s)}\left(\begin{array}{c}
i+s-l \\
(i+s-l) / 2
\end{array}\right) .
\end{aligned}
$$

We note that $c_{i, j, k}^{k}=0$ for $k<2$.

We rearrange the order of $r, z$ in $g_{i}$ for $i=1,2$. Thus we have

$$
\begin{aligned}
g_{1} & =\frac{1}{\sqrt{1-r^{2}}} \sum_{m=0}^{n} r^{m} \sum_{\substack{v=0 \\
m-v \text { even }}}^{m} r^{-v} \sum_{k=m}^{n} z^{k-m} \sum_{\substack{s=0 \\
s \text { even }}}^{v} c_{v-s, m-v+s, k-m}^{k} d_{s, m-v+s} \\
& =\frac{1}{\sqrt{1-r^{2}}} \sum_{\substack{w=0 \\
w \text { even }}}^{n} r^{w} \sum_{k=w}^{n} \sum_{v=0}^{k-w} z^{k-w-v} \sum_{\substack{s=0 \\
\text { seven }}}^{v} c_{v-s, w+s, k-w-v}^{k} d_{s, w+s}
\end{aligned}
$$




$$
=\frac{1}{\sqrt{1-r^{2}}} \sum_{\substack{w=0 \\ w \text { even }}}^{n} r^{w} \sum_{\alpha=w}^{n} z^{\alpha-w} \sum_{v=0}^{n-\alpha} \sum_{\substack{s=0 \\ s \text { even }}}^{v} c_{v-s, w+s, \alpha-w}^{\alpha+v} d_{s, w+s} .
$$

In the previous first equality $v=i+s$, in the second one $w=m-v$, and finally in the third $\alpha=k-v$. Moreover,

$$
\begin{aligned}
& g_{2}=\sum_{m=0}^{n} r^{m} \sum_{k=m}^{n} z^{k-m} \sum_{\substack{i=0 \\
m-i \text { even }}}^{m} c_{i, m-i, k-m}^{k} \sum_{\substack{\delta=i \\
\delta-i \text { even }}}^{m} d_{\delta-i, m-i} \sum_{\substack{l>0 \\
\delta-l \text { even }}}^{\delta} e_{\delta, l} r^{-l} \\
& =\sum_{m=0}^{n} r^{m} \sum_{k=m}^{n} z^{k-m} \sum_{\substack{l>0 \\
m-l \text { even }}}^{m} r^{-l} \sum_{\substack{\delta=l \\
\delta-l \text { even }}}^{m} \sum_{\substack{i=0 \\
\delta-i \text { even }}}^{\delta} d_{\delta-i, m-i} e_{\delta, l} c_{i, m-i, k-m}^{k} \\
& =\sum_{\substack{w=0 \\
w \text { even }}}^{n} r^{w} \sum_{k=w}^{n} \sum_{l>0}^{k-w} z^{k-w-l} \sum_{\substack{\delta=l \\
\delta-l \text { even } \delta-i \text { even }}}^{w+l} \sum_{\begin{array}{c}
i=0 \\
\delta
\end{array}}^{\delta} d_{\delta-i, w+l-i} e_{\delta, l} c_{i, w+l-i, k-w-l}^{k} \\
& =\sum_{\substack{w=0 \\
w \text { even }}}^{n} r^{w} \sum^{n} z^{\alpha-w} \sum_{l>0}^{n-\alpha} \sum_{\substack{u=0 \\
u \text { even } \\
u-i \text { even }}}^{w} \sum_{\substack{i=0 \\
l+l}}^{u+l} u+i, w+l-i e_{u+l, l} c_{i, w+l-i, \alpha-w}^{\alpha+l} .
\end{aligned}
$$

In the previous first equality $\delta=i+s$, in the second one $w=m-l$, in the third $\alpha=k-l$, and finally in the fourth $u=\delta-l$.

Defining $t=\sqrt{1-r^{2}}$ we have

$$
\begin{aligned}
\sqrt{1-r^{2}} g_{1}(r, z) & =t g_{1}(t, z) \\
& =\sum_{\substack{w=0 \\
w \text { even }}}^{n}\left(1-t^{2}\right)^{\frac{w}{2}} \sum_{\alpha=w}^{n} z^{\alpha-w} g_{w, \alpha}^{1} \\
& =\sum_{\substack{w=0 \\
w \text { even }}}^{n} \sum_{\substack{\rho=0 \\
\rho \text { even }}}^{w}(-1)^{\frac{\rho}{2}}\left(\begin{array}{c}
\frac{w}{2} \\
\frac{\rho}{2}
\end{array}\right) t^{\rho} \sum_{\alpha=w}^{n} z^{\alpha-w} g_{w, \alpha}^{1} \\
& =\sum_{\substack{\rho=0 \\
\rho \text { even }}}^{n} t^{\rho} \sum_{\xi=0}^{n-\rho} z^{\xi} \sum_{\substack{w=\rho \\
w \text { even }}}^{n-\xi}(-1)^{\frac{\rho}{2}}\left(\begin{array}{c}
\frac{w}{2} \\
\frac{\rho}{2}
\end{array}\right) g_{w, w+\xi}^{1},
\end{aligned}
$$

in the last equality we have $\xi=\alpha-w$, and

$$
g_{2}(r, z)=g_{2}(t, z)=\sum_{\substack{\rho=0 \\
\rho \text { even }}}^{n} t^{\rho} \sum_{\xi=0}^{n-\rho} z^{\xi} \sum_{\substack{w=\rho \\
w \text { even }}}^{n-\xi}(-1)^{\frac{\rho}{2}}\left(\begin{array}{c}
\frac{w}{2} \\
\frac{\rho}{2}
\end{array}\right) g_{w, w+\xi}^{2},
$$


where

$$
\begin{aligned}
& g_{w, w+\xi}^{1}=\sum_{v=0}^{n-w-\xi} \sum_{\substack{s=0 \\
s \text { even }}}^{v} c_{v-s, w+s, \xi}^{w+\xi+v} d_{s, w+s}, \\
& g_{w, w+\xi}^{2}=\sum_{l>0}^{n-w-\xi} \sum_{\substack{u=0 \\
u \text { even }}}^{w} \sum_{\substack{i=0 \\
l-i \text { even }}}^{u+l} d_{u+l-i, w+l-i} e_{u+l, l} c_{i, w+l-i, \xi}^{w+\xi+l}
\end{aligned}
$$

We have $g(r, z)=(t, z)=\frac{1}{t} P(t, z)$, where $P(t, z)=c z+\sum_{\rho=0}^{n+1} P_{\rho}(z) t^{\rho}$ and

(10) $P_{\rho}(z)= \begin{cases}\sum_{\xi=0}^{n-\rho} z^{\xi} \sum_{\substack{w=\rho \\ w \text { even }}}^{n-\xi}(-1)^{\frac{\rho}{2}}\left(\begin{array}{c}\frac{w}{2} \\ \frac{\rho}{2}\end{array}\right) g_{w, w+\xi}^{1} & \text { if } \rho \text { even } \\ \sum_{\xi=0}^{n-\rho+1} z^{\xi} \sum_{\substack{w=\rho-1 \\ w \text { even }}}^{n-\xi}(-1)^{\frac{\rho-1}{2}}\left(\begin{array}{c}\frac{w}{2} \\ \frac{\rho-1}{2}\end{array}\right) g_{w, w+\xi}^{2} & \text { if } \rho \text { odd }\end{cases}$

In fact $P(t, z)$ is a polynomial of degree $n$ in the variables $t$ and $z$, respectively, which can be written as

(11) $P= \begin{cases}c z+P_{n}^{0}+\hat{P}_{n-1}^{1} t+P_{n-2}^{2} t^{2}+\hat{P}_{n-3}^{3} t^{3}+\cdots+P_{0}^{n} t^{n} & n \text { even, } \\ c z+P_{n}^{0}+\hat{P}_{n-1}^{1} t+P_{n-2}^{2} t^{2}+\hat{P}_{n-3}^{3} t^{3}+\cdots+\hat{P}_{0}^{n} t^{n} & n \text { odd, }\end{cases}$

where the polynomials $P_{\zeta}^{\rho}=P_{\zeta}^{\rho}(z)$ or $\hat{P}_{\zeta}^{\rho}=\hat{P}_{\zeta}^{\rho}(z)$ of degree $\zeta$ denote the coefficient of $t^{\rho}$. Then, we expand it and arrange the coefficients of $z^{m} t^{\rho}$ into the following table:

\begin{tabular}{|c|c|c|c|c|l|c|c|}
\hline$n$ & $n-1$ & $n-2$ & $n-3$ & $n-4$ & $\cdots$ & 0 & $m / \rho$ \\
\hline$P_{n, n}^{0}$ & $P_{n, n-1}^{0}$ & $P_{n, n-2}^{0}$ & $P_{n, n-3}^{0}$ & $P_{n, n-4}^{0}$ & $\cdots$ & $P_{n, 0}^{0}$ & 0 \\
\hline$\hat{P}_{n, n}^{1}$ & $\hat{P}_{n, n-1}^{1}$ & $\hat{P}_{n, n-2}^{1}$ & $\hat{P}_{n, n-3}^{1}$ & $\hat{P}_{n, n-4}^{1}$ & $\cdots$ & $\hat{P}_{n, 0}^{1}$ & 1 \\
\hline & & $P_{n-2, n-2}^{2}$ & $P_{n-2, n-3}^{2}$ & $P_{n-2, n-4}^{2}$ & $\cdots$ & $P_{n-2,0}^{2}$ & 2 \\
\hline & & $\hat{P}_{n-2, n-2}^{3}$ & $\hat{P}_{n-2, n-3}^{3}$ & $\hat{P}_{n-2, n-4}^{3}$ & $\cdots$ & $\hat{P}_{n-2,0}^{3}$ & 3 \\
\hline & & & & $\ldots$ & $\cdots$ & $\cdots$ & $\vdots$ \\
\hline
\end{tabular}


where

$$
\begin{aligned}
P_{n-\rho, \xi}^{\rho} & =\sum_{\substack{w=\rho \\
w \text { even }}}^{n-\xi} \sum_{v=0}^{n-\xi-w} \sum_{\substack{s=0 \\
\text { seven }}}^{v} * c_{v-s, w+s, \xi}^{\xi+w+v} \\
& =\sum_{\substack{w=\rho \\
w \text { even }}}^{n-\xi} \sum_{\substack{v=0 \\
\text { even }}}^{n-\xi-w} \sum_{\substack{i=0 \\
v-i \text { even }}}^{v} * c_{i, w+v-i, \xi}^{\xi+w+v}, \\
\hat{P}_{n-\rho, \xi}^{\rho+1} & =\sum_{\substack{w=\rho \\
w \text { even }}}^{n-\xi} \sum_{l>0}^{n-\xi-w} \sum_{\substack{u=0 \\
u \text { even }}}^{w} \sum_{\substack{i=0 \\
l-i \text { even }}}^{u+l} * c_{i, w+l-i, \xi}^{\xi+w+l} \\
& =\sum_{\substack{w=\rho \\
w \text { even }}}^{n-\xi} \sum_{l>0}^{n-\xi-w}\left(\sum_{\substack{i=0 \\
l-i \text { even }}}^{l} c_{i, w+l-i, \xi}^{\xi+w+l} * \sum_{\substack{i>l \\
\text { i-l even }}}^{l+w} c_{i, w+l-i, \xi}^{\xi+w+l} *\right),
\end{aligned}
$$

where $\rho$ is even and the $*$ 's denote some constants. From the formula of $\hat{P}_{n-\rho, \xi}^{\rho+1}$, it is easy to calculate that $\hat{P}_{n-\rho, n-\rho}^{\rho+1}=0$, which means that $P(t, z)$ is a polynomial in the variables of $t$ and $z$ of degree $n$, and of degree $n$ in $t$ or $z$, respectively.

In the following we consider the function $f=f(r, z)$. Taking $G(x, y, z) \equiv$ 0 we have

$$
\begin{aligned}
f= & a r+\sum_{k=2}^{n} \sum_{m=0}^{k} r^{m} z^{k-m} \sum_{\substack{i+j=m \\
j \text { even }}} a_{i, j, k-m}^{k} I_{i+1, j} \\
= & a r+\sum_{m=0}^{n} r^{m} \sum_{k=m}^{n} z^{k-m} \sum_{\substack{i=0 \\
m-i \text { even }}}^{m} a_{i, m-i, k-m}^{k} I_{i+1, m-i} \\
& \quad-\sum_{m=0}^{1} r^{m} \sum_{m \leq k<2} z^{k-m} \sum_{\substack{i=0 \\
m-i \text { even }}}^{m} a_{i, m-i, k-m}^{k} I_{i, m-i} \\
= & a r+f_{1}(r, z)+f_{2}(r, z),
\end{aligned}
$$

where

$$
\begin{aligned}
& f_{1}=\sum_{m=0}^{n} r^{m} \sum_{k=m}^{n} z^{k-m} \sum_{\substack{i=0 \\
m-i \text { even }}}^{m} a_{i, m-i, k-m}^{k} \sum_{\substack{s=0 \\
s \text { even }}}^{m-i} A_{s, m-i} \frac{r^{-(i+s+1)}}{\sqrt{1-r^{2}}}, \\
& f_{2}=\sum_{m=0}^{n} r^{m} \sum_{k=m}^{n} z^{k-m} \sum_{\substack{i=0 \\
m-i \text { even }}}^{m} a_{i, m-i, k-m}^{k-i} \sum_{\substack{s=0 \\
s \text { even }}}^{m} A_{s, m-i} \sum_{\substack{l=0 \\
i-\text { leven }}}^{i+s} E_{i+s, l} r^{-l-1},
\end{aligned}
$$


where

$$
\begin{aligned}
A_{s, m-i} & =(-1)^{s / 2}\left(\begin{array}{c}
(m-i) / 2 \\
s / 2
\end{array}\right), \\
E_{i+s, l} & =(-1)^{l} 2^{l-(i+s)}\left(\begin{array}{c}
i+s-l \\
(i+s-l) / 2
\end{array}\right) .
\end{aligned}
$$

Note that $A_{s, m-i}$ and $E_{i+s, l}$ are the same as $d_{s, m-i}$ and $e_{i+s, l}$, we also use them later on. Moreover, note that $a_{i, j, k}^{k}=0$ for $k<2$.

We rearrange the order of $r, z$ in $f_{i}, i=1,2$, thus we have

$$
\begin{aligned}
f_{1} & =\frac{1}{r \sqrt{1-r^{2}}} \sum_{m=0}^{n} r^{m} \sum_{\substack{v=0 \\
m-v \text { even }}}^{m} r^{-v} \sum_{k=m}^{n} z^{k-m} \sum_{\substack{s=0 \\
s \text { even }}}^{v} a_{v-s, m-v+s, k-m}^{k} d_{s, m-v+s} \\
& =\frac{1}{r \sqrt{1-r^{2}}} \sum_{\substack{w=0 \\
w \text { even }}}^{n} r^{w} \sum_{k=w}^{n} \sum_{v=0}^{k-w} z^{k-w-v} \sum_{\substack{s=0 \\
s \text { even }}}^{v} a_{v-s, w+s, k-w-v}^{k} d_{s, w+s} \\
& =\frac{1}{r \sqrt{1-r^{2}}} \sum_{\substack{w=0 \\
w \text { even }}}^{n} r^{w} \sum_{\alpha=w}^{n} z^{\alpha-w} \sum_{v=0}^{n-\alpha} \sum_{\substack{s=0 \\
s \text { even }}}^{v} a_{v-s, w+s, \alpha-w}^{\alpha+v} d_{s, w+s},
\end{aligned}
$$

In the previous first equality $v=i+s$, in the second one $w=m-v$, and finally in the third $\alpha=k-v$. Moreover,

$$
\begin{aligned}
& f_{2}=\frac{1}{r} \sum_{m=0}^{n} r^{m} \sum_{k=m}^{n} z^{k-m} \sum_{\substack{i=0 \\
m-i \text { even }}}^{m} a_{i, m-i, k-m}^{k} \sum_{\substack{\delta=i \\
\delta-i \text { even }}}^{m} d_{\delta-i, m-i} \sum_{\substack{l=0 \\
\delta-l \text { even }}}^{\delta} e_{\delta, l} r^{-l}
\end{aligned}
$$

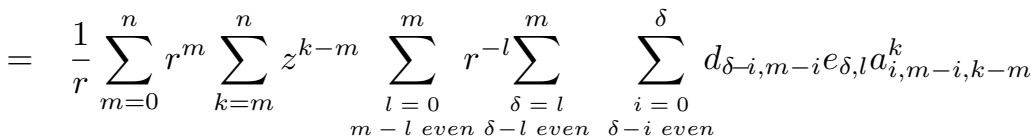

$$
\begin{aligned}
& =\frac{1}{r} \sum_{\substack{w=0 \\
w \text { even }}}^{n} r^{w} \sum_{k=w}^{n} \sum_{l=0}^{k-w} z^{k-w-l} \sum_{\substack{\delta=l \\
\delta-l \text { even }}}^{w+l} \sum_{\substack{i=0 \\
\delta-i \text { even }}}^{\delta} d_{\delta-i, w+l-i} e_{\delta, l} a_{i, w+l-i, k-w-l}^{k} \\
& =\frac{1}{r} \sum_{\substack{w=0 \\
w \text { even }}}^{n} r^{w} \sum_{\alpha=w}^{n} z^{\alpha-w} \sum_{l=0}^{n-\alpha} \sum_{\substack{u=0 \\
u \text { even }}}^{w} \sum_{\substack{i=0 \\
l-i \text { even }}}^{u+l} d_{u+l-i, w+l-i} e_{u+l, l} a_{i, w+l-i, \alpha-w}^{\alpha+l},
\end{aligned}
$$

In the previous first equality $\delta=i+s$, in the second one $w=m-l$, in the third $\alpha=k-l$, and finally in the fourth $u=\delta-l$. Taking $t=\sqrt{1-r^{2}}$ we 
obtain

$$
\begin{aligned}
r \sqrt{1-r^{2}} f_{1}(r, z) & =r t f_{1}(t, z) \\
& =\sum_{\substack{w=0 \\
w \text { even }}}^{n}\left(1-t^{2}\right)^{\frac{w}{2}} \sum_{\alpha=w}^{n} z^{\alpha-w} f_{w, \alpha}^{1} \\
& =\sum_{\substack{w=0 \\
w \text { even }}}^{n} \sum_{\substack{\rho=0 \\
\rho \text { even }}}^{w}(-1)^{\frac{\rho}{2}}\left(\begin{array}{c}
\frac{w}{2} \\
\frac{\rho}{2}
\end{array}\right) t^{\rho} \sum_{\alpha=w}^{n} z^{\alpha-w} f_{w, \alpha}^{1} \\
& =\sum_{\substack{\rho=0 \\
\rho \text { even }}}^{n} t^{\rho-\rho} \sum_{\xi=0}^{n-\xi} \sum_{\substack{w=\rho \\
w \text { even }}}^{n-\xi}(-1)^{\frac{\rho}{2}}\left(\begin{array}{c}
\frac{w}{2} \\
\frac{\rho}{2}
\end{array}\right) f_{w, w+\xi}^{1},
\end{aligned}
$$

in the last equality we have $\xi=\alpha-w$, and

$$
r f_{2}(r, z)=r f_{2}(t, z)=\sum_{\substack{\rho=0 \\
\rho \text { even }}}^{n} t^{\rho} \sum_{\xi=0}^{n-\rho} z^{\xi} \sum_{\substack{w=\rho \\
w \text { even }}}^{n-\xi}(-1)^{\frac{\rho}{2}}\left(\begin{array}{c}
\frac{w}{2} \\
\frac{\rho}{2}
\end{array}\right) f_{w, w+\xi}^{2}
$$

where

$$
\begin{aligned}
& f_{w, w+\xi}^{1}=\sum_{v=0}^{n-\xi-w} \sum_{\substack{s=0 \\
s \text { even }}}^{v} a_{v-s, w+s, \xi}^{\xi+w+v} d_{s, w+s} \\
& =\sum_{v=0}^{n-\xi-w} \sum_{\substack{i=0 \\
v-i \text { even }}}^{v} a_{i, w+v-i, \xi}^{\xi+w+v} d_{v-i, w+v-i}, \\
& f_{w, w+\xi}^{2}=\sum_{l=0}^{n-\xi-w} \sum_{\substack{u=0 \\
u \text { even }}}^{w} \sum_{\substack{i=0 \\
l-i \text { even }}}^{u+l} d_{u+l-i, w+l-i} e_{u+l, l} a_{i, w+l-i, \xi}^{\xi+w+l} \\
& =\sum_{l=0}^{n-\xi-w}\left(\sum_{\substack{i=0 \\
l-i \text { even }}}^{l} * a_{i, w+l-i, \xi}^{\xi+w+l}+\sum_{\substack{i>l \\
l-i \text { even }}}^{l+w} * a_{i, w+l-i, \xi}^{\xi+w+l}\right),
\end{aligned}
$$

where the *'s denote some constants. We have $f(r, z)=f(t, z)=$ $\frac{1}{t \sqrt{1-t^{2}}} Q(t, z)$, where $Q(t, z)=a\left(1-t^{2}\right)+\sum_{\rho=0}^{n+1} Q_{\rho}(z) t^{\rho}$ and

$(12)$

$$
Q_{\rho}(z)= \begin{cases}\sum_{\xi=0}^{n-\rho} z^{\xi} \sum_{\substack{w=\rho \\
w \text { even }}}^{n-\xi}(-1)^{\frac{\rho}{2}}\left(\begin{array}{c}
\frac{w}{2} \\
\frac{\rho}{2}
\end{array}\right) f_{w, w+\xi}^{1} & \text { if } \rho \text { even, } \\
\sum_{\xi=0}^{n-\rho+1} z^{\xi} \sum_{\substack{w=\rho-1 \\
\text { weven }}}^{n-\xi}(-1)^{\frac{\rho-1}{2}}\left(\begin{array}{c}
\frac{w}{2} \\
\frac{\rho-1}{2}
\end{array}\right) f_{w, w+\xi}^{2} & \text { if } \rho \text { odd. }\end{cases}
$$


In fact $Q(t, z)$ is a polynomial in the variables of $t$ and $z$ of degree $n+1$, of degree $n$ in $z$, which can be written as

$$
Q= \begin{cases}a\left(1-t^{2}\right)+Q_{n}^{0}+\hat{Q}_{n}^{1} t+Q_{n-2}^{2} t^{2}+\cdots+\hat{Q}_{0}^{n+1} t^{n+1} & n \text { even } \\ a\left(1-t^{2}\right)+Q_{n}^{0}+\hat{Q}_{n}^{1} t+Q_{n-2}^{2} t^{2}+\cdots+\hat{Q}_{1}^{n} t^{n} & n \text { odd }\end{cases}
$$

where the polynomials $Q_{\zeta}^{\rho}=Q_{\zeta}^{\rho}(z)$ or $\hat{Q}_{\zeta}^{\rho}=\hat{Q}_{\zeta}^{\rho}(z)$ of degree $\zeta$ denote the coefficient of $t^{\rho}$.

Moreover, from (7), we have $f(0, z)=0$ which implies $Q(1, z)=0$, i.e. $Q(t, z)=(t-1) \bar{Q}(t, z)$, where $\bar{Q}(t, z)$ is a polynomial in the variables of $t$ and $z$ of degree $n$, and of degree at most $n$ in $t$ or $z$, respectively. Hence, by Bezout's Theorem the maximum number of the common solution of $P(t, z)$ and $Q(t, z)$ is at most $n^{2}$ for $0<t<1$, because $P(t, z)$ and $\bar{Q}(t, z)$ are the polynomials in the variables of $t$ and $z$ of degree at most $n$, respectively. Thus, by Theorem (2), the maximum number of limit cycles bifurcating form system (1) is $n^{2}$.

Next, we shall provide a system having $n^{2}$ limit cycles. Here, we just consider $n$ even, and we take $G(x, y, z)=0$ and

$$
\begin{aligned}
& F(x, y, z)=\sum_{k=2}^{n} a_{0,0, k}^{k} z^{k}+a_{1,0,1}^{2} x z \\
& R(x, y, z)=\sum_{\substack{k=2 \\
k \text { even }}}^{n}\left(c_{k, 0,0}^{k} x^{k}+c_{0, k, 0}^{k} y^{k}\right) .
\end{aligned}
$$

Computing the averaged functions and taking $t=\sqrt{1-r^{2}}$, we have

$$
\begin{aligned}
r \sqrt{1-r^{2}} f(r, z) & =a r^{2}+\left(a_{1,0,1}^{2} z-\sum_{k=2}^{n} a_{0,0, k}^{k} z^{k}\right)\left(1-\sqrt{1-r^{2}}\right) \\
& =(1-t)\left(a(1+t)+a_{1,0,1}^{2} z-\sum_{k=2}^{n} a_{0,0, k}^{k} z^{k}\right) \\
& =(1-t)(a(1+t)-\bar{Q}(z)),
\end{aligned}
$$

where $\bar{Q}(z)$ is an arbitrary polynomial in $z$ of degree $n$, such that $\bar{Q}(0)=0$. At the same time, the averaged function corresponding to $R(x, y, z)$ satisfies

$$
\sqrt{1-r^{2}} g(r, z)=c z+\sum_{\substack{k=2 \\ k \text { even }}}^{n} r^{k}\left(c_{k, 0,0}^{k} I_{k, 0}+c_{0, k, 0}^{k} I_{0, k}\right)
$$


Using (4) we obtain that $\sqrt{1-r^{2}} g(r, z)=c z+g_{1}(r)+g_{2}(r)$, where

$$
\begin{aligned}
& g_{1}=\sum_{\substack{k=2 \\
k \text { even }}}^{n} c_{k, 0,0}^{k}-\sqrt{1-r^{2}} \sum_{\substack{m=0 \\
m \text { even }}}^{n-1} r^{m} \sum_{\substack{k=m+1 \\
k \text { even }}}^{n} c_{k, 0,0}^{k} 2^{-m}\left(\begin{array}{c}
m \\
\frac{m}{2}
\end{array}\right), \\
& g_{2}=\sum_{\substack{m=0 \\
m \text { even }}}^{n} A_{m} r^{m}+\sqrt{1-r^{2}} \sum_{\substack{m=0 \\
m \text { even }}}^{n-1} B_{m} r^{m},
\end{aligned}
$$

with

$$
A_{m}=\sum_{\substack{k=m \\ k \text { even }}}^{n} c_{0, k, 0}^{k} d_{k-m, k}, \quad B_{m}=\sum_{\substack{k=m \\ k \text { even }}}^{n} c_{0, k, 0}^{k} d_{k-m, k} \sum_{\substack{l>0 \\ l \text { even }}}^{n} e_{k-m, l} .
$$

Writing $t=\sqrt{1-r^{2}}$ from the definition (7) the polynomials $P_{i}(t)=g_{i}(r)$, $i=1,2$, satisfy $P_{i}(1)=g_{i}(0)=0$. Then we can define a polynomial in $t$ of degree $n, \bar{P}(t)=P_{1}(t)+P_{2}(t)=(t-1) \tilde{P}(t)$. We claim that $\bar{P}(t)$ is an arbitrary polynomial such that $\bar{P}(1)=0$. It is obvious to know that $g_{1}, g_{2}$ have $n / 2$ parameters, respectively, where the $n / 2$ coefficients $c_{0, k, 0}^{k}$ allow to choose the first term of $g_{2}$ arbitrarily but the term with $m=0$, implying that the even terms of $\bar{P}(t)$ are arbitrary but the constant term; while another $n / 2$ coefficients $c_{k, 0,0}^{k}$ allow to choose the second term in $g_{1}$ arbitrarily, implying that the odd terms of $\bar{P}(t)$ are arbitrary. So, the polynomial $\bar{P}(t)$ of degree $n$ satisfies $\bar{P}(1)=0$, and has $n$ arbitrary coefficients, which completes our claim. ( see also [2])

In short, the number of the solutions of $f(r, z)=0, g(r, z)=0$ is equal to the number of the intersection points of the curves

$$
\ell_{1}: \quad c z+\bar{P}(t)=0, \quad \ell_{2}: \quad a(1+t)-\bar{Q}(z)=0 .
$$

Proposition 6. System (15) has at least $n^{2}$ common solutions $(t, z)$ in $(0,1) \times\left(0, z^{*}\right)$ for any given positive number $z^{*}$.

Proof We know that the point $(t, z)=(-1,0)$ lies on the curve $\ell_{2}$. Hence, we can choose some suitable coefficients in (14), such that for any given positive number $z^{*}>0, \ell_{2}$ intersects the line $t=0 n$ times in the interval $\left(0, z^{*}\right)$, and the smallest maximum,

$$
\hat{t}=\min \left\{t=\bar{Q}(z) / a-1 \mid \bar{Q}^{\prime}(z)=0, \bar{Q}^{\prime \prime}(z) / a<0, z \in\left(0, z^{*}\right)\right\},
$$

is larger than 1 . Hence, there are $n$ intersection points between $\ell_{2}$ and $t=\bar{t}$ for all $\bar{t} \in(0,1)$. 
On the other hand, we know that the point $(1,0)$ lies on the curve $\ell_{1}$. Hence, we take $\ell_{1}$, such that it intersects the line $z=0 n$ times in the interval $(0,1]$ and the smallest maximum,

$$
\hat{z}=\min \left\{z=-\bar{P}(t) / c \mid \bar{P}^{\prime}(z)=0,-\bar{P}^{\prime \prime}(t) / c<0, t \in(0,1)\right\},
$$

is larger than $z^{*}$. Hence, there are $n^{2}$ intersection points between $\ell_{1}$ and $\ell_{2}$ contained in the rectangle $(0,1) \times\left(0, z^{*}\right)$.

We can consider the case $n$ odd in a similar way. Provided that we take in (14),

$$
R(x, y, z)=c_{n, 0,0}^{n} x^{n}+\sum_{\substack{k=2 \\ k \text { even }}}^{n}\left(c_{k, 0,0}^{k} x^{k}+c_{0, k, 0}^{k} y^{k}\right),
$$

we can get the same curves (15) and the same result.

The proof of Proposition 6 implies that for any integer $n>2$, we can find $n^{2}$ intersection points on $f(r, z)=0$ with $g(r, z)=0$ for $r \in\left(r_{0}, 1\right)$, $0<r_{0} \ll 1$, which (using the averaging theory, see Theorem 2) give rise to $n^{2}$ limit cycles bifurcating from the periodic orbits of the system $\dot{x}=-y(1+x), \dot{y}=x(1+x), \dot{z}=0$.

Furthermore, if $G(x, y, z) \not \equiv 0$, then we can know that $Q(t, z)$ in $(13)$ is also a polynomial in the variables $t$ and $z$ of degree $n+1$, and of degree at most $n$ in $z$. And we also have $Q(1, z)=0$, which implies $Q(t, z)=$ $(t-1) \bar{Q}(t, z)$, where $\bar{Q}(t, z)$ is a polynomial in the variables $t$ and $z$ of degree $n$. Hence, by Bezout's Theorem the maximum number of the common solution of $P(t, z)$ and $Q(t, z)$ is at most $n^{2}$ for $0<t<1$. Thus, the maximum number of limit cycles bifurcating form system (1) is $n^{2}$, if we use the averaging theory up to first order in $\varepsilon$.

\section{ACKNOWLEDGEMENTS}

The first author is partially supported by a DGICYT grant number MTM2005-06098-C02-01 and by a CICYT grant number 2005SGR 00550. The second author has been supported partly by Grants NSFC-10371072 of China, and Grant G63009138 of Spain. He thanks to CRM and to Department of Mathematics of the Universitat Autònoma de Barcelona for their support and hospitality.

\section{REFERENCES}

[1] A. Cima, J. Llibre And M.A. Teixeira, Limit cycles of some polynomial differential systems in dimension 2, 3 and 4 via averaging theory, preprint, 2005.

[2] J. Llibre, J.S. Río AND J.A. RodrígueZ, Averaging analysis of a perturbated quadratic center, Nonlinear Anal. 46 (2001), 45-51. 
[3] J.A. Sanders and F. Verhulst, Averaging methods in nonlinear dynamical systems, Applied Mathematical Sci., Vol. 59, Springer-Verlag, New York, 1985.

[4] F. VerhUlSt, Nonlinear differential equations and dynamical systems, Universitext. Springer-Verlag, Berlin, 1996

[5] H ŻOŁA̧DEK, Limit cycles appearing after perturbation of certain multidimensional vector fields, J. Dynam. Differential Equations 13 (2001), 689-709.

[6] H ŻOŁA̧DEK, Limit cycles for multidimensional vector fields. The elliptic case, J. Dynam. Control Systems 9 (2003), 265-310.

[7] H ŻOŁA̧DEK, Limit cycles of three-dimensional polynomial vector fields, Nonlinearity 18 (2005), 175-209.

1 Departament de Matemàtiques, Universitat Autònoma de Barcelona, 08193 Bellaterra, Barcelona, Spain

E-mail address: jllibre@mat.uab.es

2 Department of Mathematics, Shanghai Jiaotong University, Shanghai, 200240, CHINA

E-mail address: jiangyu@sjtu.edu.cn 